\title{
Towards a Seamless Future Generation Network for High Speed Wireless Communications
}

\author{
Kelvin O. O. Anoh ${ }^{1}$, Raed A. A. Abd-Alhameed ${ }^{1}$, Michael Chukwu' ${ }^{2}$, Mohammed Buhari ${ }^{2}$ and Steve M. R. Jones ${ }^{1}$ \\ ${ }^{1}$ Mobile and Satellite Communications Research Centre, University of Bradford (MSCRC), BD1 7DP - United Kingdom \\ ${ }^{2}$ Centre for Satellite Technology Development, National Space Research Development Agency, Abuja - Nigeria
}

\begin{abstract}
The MIMO technology towards achieving future generation broadband networks design criteria is presented. Typical next generation scenarios are investigated. The MIMO technology is integrated with the OFDM technology for effective space, time and frequency diversity exploitations for high speed outdoor environment. Two different OFDM design kernels (fast Fourier transform (FFT) and wavelet packet transform (WPT)) are used at the baseband for OFDM system travelling at terrestrial high speed for $800 \mathrm{MHz}$ and $2.6 \mathrm{GHz}$ operating frequencies. Results show that the wavelet kernel for designing OFDM systems can withstand doubly selective channel fading for mobiles speeds up to $280 \mathrm{Km} / \mathrm{hr}$ at the expense of the traditional OFDM design kernel, the fast Fourier transform.
\end{abstract}

Keywords-Doppler Effect; Doubly selective fading; frequencyselective fading; OFDM; Wavelet; MIMO

\section{INTRODUCTION}

The broadband network for next generation communications is a multiple input multiple output (MIMO) network [1-3] defined around $2 \times 2,4 \times 2$ and $4 \times 4$ MIMO systems such as the long-term evolution (LTE) and LTE advanced [4]. It uses OFDM for the downlink [5, 6]. The broadband LTE-advanced Release 10, for instance, is now greatly being pursued for building more dependable seamless service and was earlier believed to offer stationary downlink (DL) data size of $1 \mathrm{~Gb} / \mathrm{s}$ and mobile data size of $100 \mathrm{Mb} / \mathrm{s}$ in wireless communications [7], although it has been extended now to $3 \mathrm{~Gb} / \mathrm{s}$ DL in the subsequent Releases [8]. The airinterface of this technology adopts the OFDM since it can combat inter-symbol interference (ISI) and also converts the frequency selective channel into a flat-fading channel. Also, OFDM is the key air-interface in digital video broadcasting for -terrestrial (DVB-T) and -satellite (DVB-S) wireless communications. Thus, the broadband LTE-advanced uses MIMO-OFDM.

One of the most advocated methods of deploying multiantenna systems in broadband technology involve the spacetime coding [1, 2]. It is the MIMO standard for LTEAdvanced [9]. This is because STBC can exploit the space, time and frequency diversity gains. Most early space-time (ST) coding scheme discuss the case of a frequency-flat fading channel. In practice, the broadband wireless communication channel is frequency-selective and exhibit inter-symbol interference (ISI) that depletes the performance of the system [10]. For broadband wireless systems, the MIMO channels experience frequency-selective fading, which complicates the design of ST codes because of ISI [11].
This is usually addressed by the use of OFDM with MIMO, hence the MIMO-OFDM. The combination of MIMO and OFDM provides an attractive interface solution for nextgeneration wireless local area networks (WLANS), wireless metropolitan area networks (WMAN) and fourth generation mobile cellular wireless systems [12].

For instance MIMO technology provides two design advantages, namely spatial multiplexing which provides capacity gain, and space-time coding which improves link dependability through diversity gain $[11,12]$. These advantages are usually exploited in the design of multiantenna systems. Applying ST codes only separately over each frequency tone of the OFDM does not exploit frequency diversity. Thus, we choose to construct only space-time frequency (STF) codes that can exploit space, time and frequency diversities.

Broadband network users are necessarily non-stationary. The movement of the user relative to the transmitter cause the signals to fade depending on how fast the channel varies. This is usually discussed as time-selectively (in addition to the frequency-selectivity) which is the case of a moving receiver. STBC with OFDM can also be studied as space-frequency block codes (SFBC) [13, 14] or space-time frequency block codes (STFBC) [11] over frequency selective channels [15]. The SFBC provides spatial diversity gain only, which sometimes, can be achieved by forward error correction coding with interleaving [12].

The STFBC is preferred over SFBC since the former exploits space, time and frequency gains $[10,11]$ and the later exploits only space and frequency diversities but not time [10, $13,14]$. The advantage of combining MIMO and OFDM is that MIMO provides capacity and diversity gains while OFDM mitigates the deterring multipath effect. Like this study aims to establish, a high-speed mobile on railway wireless communication technology based on LTE has been discussed in [5] for traditional OFDM architecture. However, it will be shown that the traditional OFDM system is sub-optimal for high speed wireless communications.

In Section II, the OFDM design kernels are presented using the traditional FFT and wavelet transform while the channel system model is discussed in Section III including the Doppler effects. In Section IV, the simulation environment with results are discussed which including the single-inputsingle-out (SISO) and the MIMO channel, a typical representation of the broadband channel. The conclusion follows in Section V.

*This paper has been presented in part at the CSTD

conference week on August 21-22, 2013. 


\section{ORThOGONAL FREQUENCY DIVISION MULTIPLEXING}

Orthogonal frequency division multiplexing (OFDM) divides wideband into many narrow-bands to increase the data rate. Traditionally kernel for designing OFDM is the FFT. Another kernel that provides signal representation half-way in time-frequency plane, the wavelet transform, is also discussed.

\section{A. FFT-OFDM}

OFDM system is used in baseband as modulation method that divides a selected bandwidth into smaller and many narrow bands. In time domain, an $N$-point FFT OFDM system can be represented as:

$$
s[n]=\frac{1}{\sqrt{N}} \sum_{k=0}^{N-1} S_{k} e^{j 2 \pi n k / N}, \quad n=0,1,2, \ldots, N-1
$$

where $s[n]$ is the discrete form of $s(t), N$ is the number of sub-channels, $1 / \sqrt{N}$ is a scaling factor with $n$ as the index of the prevalent subcarrier. $S_{k}$ is the QPSK mapped input symbol of $k^{\text {th }}$ sub-channel. The number of FFT points used is equivalent to the number of narrowband sub-channels over which the input symbols are multiplexed. Each of the resulting narrowband sub-channels is modulated by the mapped input bits. Cyclic prefix (CP) at least equal to the length of the channel response, $L$ is pre-appended to each OFDM symbol to combat ISI. To account for the CP, Equation 1a can be modified as:

$$
s[n]=\frac{1}{\sqrt{N}} \sum_{k=0}^{N-1} S_{k} e^{j 2 \pi n k / N}, \quad-N_{g} \leq n \leq N-1
$$

In Equation $1 \mathrm{~b}, N_{g}$ denotes the length of $\mathrm{CP}$ pre-appended to every OFDM symbol.

\section{B. Wavelet-OFDM}

The discrete wavelet transform (DWT) can be used to study multicarrier systems as in [16]. It represents signals in time-frequency domain such that the signal exists neither purely in frequency domain nor purely in time domain. For a mapped input symbol $S_{k}$ to be transformed by the DWT, the time domain output can be realized from [17];

$$
s[n]=\sum_{k} \sum_{m=0}^{M-1} S_{k, n} \varphi_{m, n}(t)
$$

where $S_{k, n}$ represents the $n^{\text {th }}$ symbol which modulates the $m^{\text {th }}$-waveform of the $k^{\text {th }}$-constellation. $\varphi_{m, n}(t)$ represents the complex orthogonal DWT basis function similar to the traditional OFDM as:

$$
\varphi_{m, n}=\left\{\begin{array}{cc}
1 & n=m \\
0 & \text { elsewhere }
\end{array}\right.
$$

where $m$ and $n$ are scales and shifts respectively. If $n$ is the index of each discrete wavelet symbol $s[n]$ of the continuous time symbol $s(t)$, then the wavelet transform is defined as [18]:

$$
\psi_{k, a}(t)=e^{i \pi t^{2}}
$$

Now, let a continuous wavelet function be expressed as:

$$
\psi_{k, a}(t)=\frac{1}{\sqrt{k}} \psi\left(\frac{t-a}{k}\right)
$$

where $k$ and $a$ are the scaling and shifting parameters respectively and $\psi($.$) is called the mother wavelet. Then, from$ Equations 5 and 4 the resulting continuous transform can be represented as:

$$
s_{C W T}(\tau, k)=\frac{1}{\sqrt{k}} \int_{-\infty}^{\infty} \exp \left[i \pi \frac{(t-\tau)^{2}}{k^{2}}\right] s(t) d t
$$

Equation 6 has the advantage of time and frequency diversities unlike the FFT transform that has only frequency diversity advantage. In fact, it has been explored that orthogonal wavelet-based OFDM is more robust to ICI and ISI problems than the FFT-based OFDM [19-21]. Meanwhile, the CP pre-appended to the OFDM symbol when using the FFT is omitted in the wavelet-based OFDM. This provides additional $25 \%$ spectral efficiency.

\section{SYSTEM MODEL}

In the following, the system architecture is discussed for the forms of OFDM kernels just discussed. Since the fading for each transmission branch in a multi-antenna system between a pair of transmit and receive antennas are usually independent, the probability that the information is detected correctly is increased [11]. Thus, the probability that the transmit signal will be decoded correctly increases with the diversity order. On that note, let there be a MIMO-OFDM system with $N_{\mathrm{T}}$ antennas and $N_{\mathrm{R}}$ receive antennas defined for $N$-subcarrier OFDM existing for each transmit antenna. Let there be also $n^{\text {th }}$ OFDM subcarrier symbol, $s_{k}^{p}(n)$, transmitted over $p^{\text {th }}$ transmit antenna at $k^{\text {th }}$ OFDM symbol duration. It can be identified that the OFDM symbol of this characteristics$s_{k}^{p}(n)-$ will exploit frequency, space and time diversity gains by the notations following the OFDM symbol, $s($.).

\section{A. General System Model}

For the FFT-based OFDM, IFFT is applied and the CP is pre-appended to each of the symbols. In the case of waveletbased OFDM, the wavelet transform is applied to the codeword and then transformed to frequency domain - to fully exploit the frequency diversity gain. The channel is entirely doubly selective (except for times at which the Doppler frequency is explicitly null), although the OFDM technology transforms the frequency-selective channel into a frequency flat fading channel.

In the receiver (after removing the $\mathrm{CP}$ for the FFT-OFDM case), the received symbol is OFDM-detransformed using FFT or forward wavelet transform as the case may be. So for each transmit symbol from one transmit antenna,

$$
R_{k}^{q}(n)=\sum_{p=1}^{N_{T}} H_{p, q}(n) S_{k}^{p}(n)+Z_{k}^{q}(n), \forall n=0,1, \Lambda, N
$$

where $R_{k}^{q}(n)$ is the $n^{\text {th }}$ received OFDM symbol with $H_{p, q}(n)$ as the frequency domain channel gain corresponding 
to $p^{\text {th }}$ transmit antenna and $q^{\text {th }}$ receive antenna respective for every subcarrier. The channel gain corresponds to the individual resolvable paths traversed by the OFDM symbols with impulse response vector:

$\boldsymbol{h}_{p, q}=\left[h_{p, q}(0), h_{p, q}(1), h_{p, q}(2), \Lambda, h_{p, q}(L-1)\right] \in C^{L \times 1}$

where $C^{L \times 1}$ is a complex matrix of $L \times 1$ dimension. The channel gains then follow as:

$$
H_{p, q}(n)=\sum_{l=0}^{L-1} h_{p, q}(l) \cdot e^{-j 2 \pi f \tau_{l}}
$$

where $e^{-j 2 \pi f \tau_{l}}$ is the DFT factor for transforming from time to frequency domain, $Z_{k}^{q}(n)$ in Equation 7 is the additive white Gaussian noise (AWGN) while $S_{k}^{p}(n)$ is the space-timefrequency (STF) codewords of the $N_{\mathrm{T}}$ transmit antenna, $N_{\mathrm{R}}$ receive antenna of $N_{\Delta}$ OFDM symbol interval. By imaging the STF in three-dimension (3D), STF will be seen as a point on that plane [15]. Like in $[22,23]$, the diversity gain will increase by the number of subcarriers used such as $N_{T} N_{\Delta} N_{R}$. For each sub-channel, the STF codeword is constructed as follows:

$$
S(n)=\left[\begin{array}{ccc}
S_{1}^{1}(n) & \Lambda & S_{1}^{N_{T}}(n) \\
\mathrm{M} & \mathrm{O} & \mathrm{M} \\
S_{N_{\Delta}}^{1}(n) & \Lambda & S_{N_{\Delta}}^{N_{T}}(n)
\end{array}\right] \in C^{N_{\Delta} \times N_{T}}
$$

In Equation 9, $S(n)$ can be taken as orthogonal space-time block code already discussed in $[24,25]$. In terms of STBC, Equation 7 can then be expressed as [26];

$$
R(n)=\sqrt{\frac{\rho}{N_{T}}} H(n) S(n)+Z(n)
$$

where $\rho$ is the SNR respective to each transmission branch (antenna), $Z$ (.) is additive noise with circular symmetry and zero mean. To exploit the STBC basic criterion, Equation 9 must be designed according to the design criteria of [25] and given as:

$$
s=\left[\begin{array}{cc}
s_{1} & s_{2} \\
-s_{2}^{*} & s_{1}^{*}
\end{array}\right]
$$

Traditionally, full diversity of an orthogonal STBC system is derived from the pairwise error probability between two different codeword matrices (usually transmitted and received), say $\boldsymbol{S}$ and $\boldsymbol{S}^{\prime}$ bounded as [26]:

$$
P\left(\boldsymbol{S} \rightarrow \boldsymbol{S}^{\prime}\right) \leq\left(\begin{array}{c}
2 r-1 \\
r
\end{array}\right)\left(\prod_{i=0}^{r} \gamma_{i}\right)^{-1}\left(\frac{\rho}{N_{T}}\right)^{-r}
$$

Equation 11 can also be used to discuss the BER statistics of space-frequency block coding (SFBC); In fact, the BER statistic of STBC and SFBC are similar [27] where the upper bound, $r$, is the rank of: $\left(\boldsymbol{S}-\boldsymbol{S}^{\prime}\right) \mathbf{R}\left(\boldsymbol{S}-\boldsymbol{S}^{\prime}\right)^{H}$ where $\mathbf{R}=\mathrm{E}\left\{H H^{H}\right\}$ is the correlation matrix of the channel matrix and $\gamma_{1}, \gamma_{2}, \ldots, \gamma_{r}$ are nonzero eigenvalues of $\left(\boldsymbol{S}-\boldsymbol{S}^{\prime}\right) \mathbf{R}\left(\boldsymbol{S}-\boldsymbol{S}^{\prime}\right)^{H}$. The superscript in $(.)^{H}$ is a Hermitian transpose operator and $H$ represents the channel matrix. Signals $\boldsymbol{S}$ and $\boldsymbol{S}^{\prime}$ are transmitted and received signals respectively. Recently, the BER statistic of the STFBC has been explored in $[15,28]$.

\section{B. Example of an orthogonal STBC - the Alamouti Code}

The traditional Alamouti STBC is followed in which the system will be assumed to be constant over two symbol periods. $s_{1}$ and $s_{2}$ are transmitted in the first symbol period, and their conjugates $\left(s_{1}^{*}\right.$ and $\left.-s_{2}^{*}\right)$ are transmitted in the second symbol period. In Figure 2, this is replaced with the FFT. In Figure 2, $s_{1}$ will be transmitted using antenna T1 and $s_{2}$ using antenna T2 in the first time period. In the second time period, the negative conjugate of $s_{2}$, i.e. $\left(-s_{2}^{*}\right)$, and the conjugate of $s_{1}$, i.e. $s_{1}^{*}$, will be transmitted over antennas T1 and $\mathrm{T} 2$ respectively.

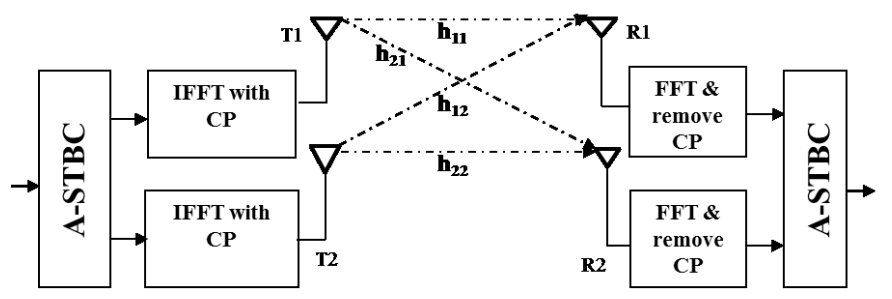

Fig. 2. Simple 2x2 MIMO-OFDM Modulation Architecture

Let $s(n)$ be the discrete equivalent of the original OFDM information $s(t)$, then $s_{1}$ and $s_{2}$ can be obtained as $[29,30]$ :

$$
\begin{aligned}
& s_{1}=\frac{1}{\sqrt{2}} s(n) \\
& s_{2}=\frac{1}{\sqrt{2}} s(n)
\end{aligned}
$$

The code structure formed from Equation 12 following Equation 9 and 10 is an STF codeword. In that case, $S, S_{1}$ and $S_{2}$ are frequency domain contents of $s, s_{1}$ and $s_{2}$ respectively. Equation 12 will convolve with the doubly selective frequency domain channel gains of $h_{1}$ and $h_{2}$, as of the channel transfer function in Equation 8b such as:

$$
R_{1}=H\left[\begin{array}{l}
S_{1} \\
S_{2}
\end{array}\right]+Z_{1}
$$

and,

$$
R_{2}=H\left[\begin{array}{r}
-S_{2}^{*} \\
S_{1}^{*}
\end{array}\right]+Z_{2}
$$

$$
\text { where } H=\left[\begin{array}{ll}
H_{1} & H_{2}
\end{array}\right]=\left[\begin{array}{ll}
h_{11} & h_{12} \\
h_{21} & h_{22}
\end{array}\right]
$$

By Equation 14, there are up to 2 number of receive antennas. The received signals estimates of the transmitted symbol will then be computed. These estimates are obtained as: 


$$
D_{1}=H_{1}^{H} R_{1}+R_{2}^{H} H_{2}
$$

and,

$$
D_{2}=H_{2}^{H} R_{1}-R_{2}^{H} H_{1}
$$

where $(.)^{H}$ denotes a Hermitian operator. The foregoing description limits $N_{\mathrm{T}}=2$ and up to $N_{\mathrm{R}}=2$.

\section{Doppler Effect in OFDM Systems - Single Antenna Case}

Consider a traditional frequency-selective channel as (in time domain) [31];

$$
h(t, \tau)=\sum_{l=0}^{L-1} \alpha(l) e^{j \phi_{l}} \delta\left(t-\tau_{l}\right)
$$

where $\alpha(l)$ is the $l^{\text {th }}$ amplitude of the $l^{\text {th }}$ path traversed by the OFDM symbol, $\phi_{l}(t)=2 \pi\left\{\left(f_{c}+f_{d, l}\right) \tau_{l}-f_{d, l} t\right\}$ is the phase, $f_{c}$ is the carrier frequency, $\tau$ is the path delay and $\delta($.$) is$ the dirac delta function. The received OFDM signal in the receiver will be;

$$
r(t, \tau)=\frac{1}{\sqrt{L T}} \sum_{l=0}^{L-1} \alpha(l) e^{j \phi_{l}} s\left(t-\tau_{l}\right)
$$

So, each of the received signals is delayed by $\tau_{l}$ and scaled by $\alpha(l) e^{j \phi_{l}}$. If frequency domain content of Equation 16 is considered as well as the doubly-selective characteristics of the channel (i.e. $t$ and $f$ ):

$$
H(t, f)=\sum_{l=0}^{L-1} h(t, \tau) e^{-j(2 \pi l) / N}
$$

Notice that $h\left(t, \tau_{l}\right) \in C^{L \times 1}$, where $C^{L \times 1}$ is a complex matrix of $L \times 1$ dimension. The term $\phi_{n}$ influences the phase rotation of received OFDM symbols and it involves $\phi_{l}=\phi_{n}+$ $\phi_{o f f}$, where $f_{d}$ is the maximum Doppler frequency and $\phi_{o f f}$ is the carrier frequency offset. In this work, so set $\phi_{\text {off }}=0$. The transmit signal convolves with Equation 18 such as;

$$
R(t, f)=\frac{1}{\sqrt{L T}} \sum_{l=0}^{L-1} H(t, f) S(f)
$$

where $S(f)$ is the frequency content of $s\left(t-\tau_{l}\right)$. The Doppler frequency shifts the signal from the carrier frequency which in turn affects the amplitude of the received symbol. For signal compensation, we proceed as follows;

$$
Y(t, f)=\frac{H(t, f)^{H} R(t, f)}{\|H(t, f)\|^{2}}
$$

where $H(t, f)^{H}$ is the Hermitian of the matrix $H(t, f)^{H}$. Equation 21 is then transformed into the time domain. If at a time that $|H(t, f)|=0$, then Equation 21 is modified as:

$$
Y(t, f)=\frac{H(t, f)^{H} R(t, f)}{\|H(t, f)\|^{2}+\varepsilon}
$$

This is equivalent to:

$$
Y(t, f)=\frac{H(t, f)^{H} \cdot H(t, f) S(t, f)}{\|H(t, f)\|^{2}+\varepsilon}+\frac{H(t, f)^{H} \cdot Z(t, f)}{\|H(t, f)\|^{2}+\varepsilon}, \forall 0 \leq \varepsilon \leq 1
$$

where $(.)^{H}$ is the Hemitian operator, $H(t, f)^{H} \cdot H(t, f)$ is unitary. The term $\varepsilon$ is an error correction factor to reduce the magnification of the noise parameter due to equalization.

\section{Doppler Effect in OFDM Systems for Multi-Antenna Case}

In earlier studies of the ST codes for MIMO systems, the channel model is usually treated as frequency flat-fading channel. However, each of the transmission branches is independent. This necessitates that the likelihood of decoding the signals correctly increases. MIMO-OFDM systems for realistic broadband channel is non-frequency flat, instead frequency-selective which the OFDM converts to flat-fading channel to eliminate ISI. Meanwhile, for varying mobile speeds the channel also varies in time contributing timeselectivity so that the broadband channel be doubly (timefrequency) selective. This is the case for each transmission channel branch in this study discussed in Section III- $A$. Thus, for the STF block codes used in this study, each of the transmission branches is necessarily doubly selective so that the Doppler frequency can be accounted for at varying mobile speeds.

\section{Simulation Results AND Discussion}

The simulation was carried out, first for the single antenna transmission and then the MIMO antennas systems. The operating frequency deployed is the new operating frequency $800 \mathrm{MHz}$ and $2.6 \mathrm{GHz}$ for LTE-advanced. The rest higher frequencies $(900 \mathrm{MHz}$ and $1800 \mathrm{MHz}$ for $\mathrm{GSM})$ were not investigated. In general, the QPSK modulation scheme was used over OFDM system whose channel delay spread is in the order of the outdoor environment at a sampling frequency of $20 \mathrm{KHz}$.

The mobile speeds are typical for standard road (and railway) travels $-120 \mathrm{Km} / \mathrm{hr}, 150 \mathrm{KM} / \mathrm{hr}, 200 \mathrm{Km} / \mathrm{hr}$, $240 \mathrm{Km} / \mathrm{hr}$ and $280 \mathrm{Km} / \mathrm{hr}$ are investigated. The number of OFDM subcarriers used is 64 with $25 \%$ cyclic prefix for the case of FFT-OFDM. This is not applicable to wavelet-OFDM by the considerations described in this work, thus offering the wavelet-OFDM further $25 \%$ spectrum efficiency at the expense of the FFT-OFDM.

\section{A. SISO OFDM System}

The SISO-OFDM systems considered are for the FFTbased and wavelet-based OFDM systems. Each has been simulated over the same design parameters except for the FFT-OFDM using cyclic prefix of $25 \%$ only. 


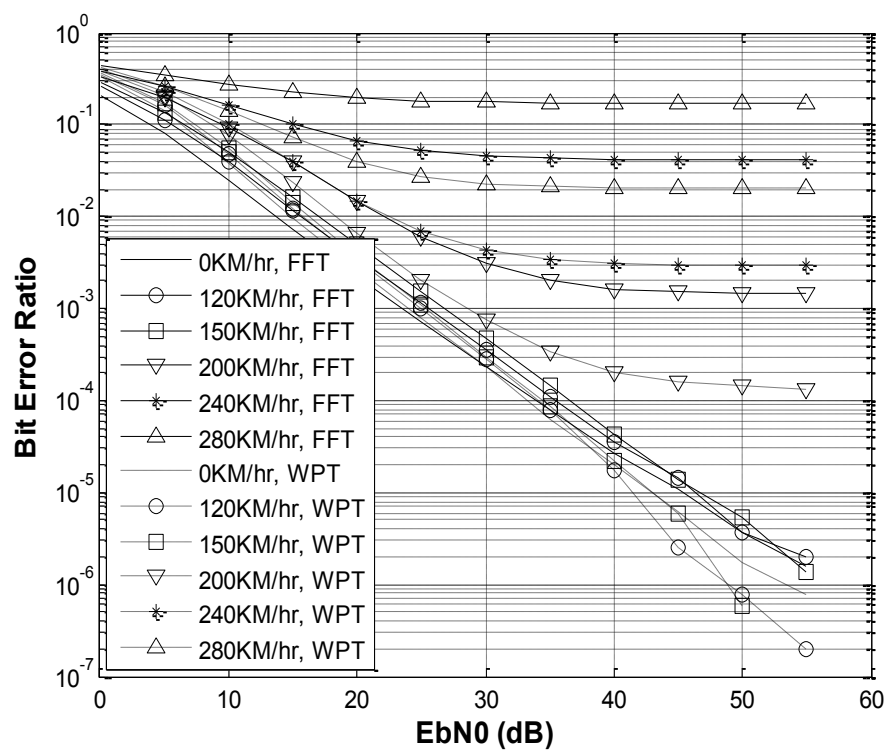

Fig. 3. Comparison of FFT-OFDM and wavelet-OFDM at $800 \mathrm{MHz}$ for SISO Systems

Recall that the FFT-OFDM is highly robust over frequency selective channel [32]; $f_{d}=0$ in that case. From Figures 3 and 4 , it can be seen that the FFT-OFDM contended favourably with the wavelet-OFDM when the channel is dominantly frequency-selective. However, for other speeds shown in the results, wavelet-OFDM well outperformed the FFT-OFDM.

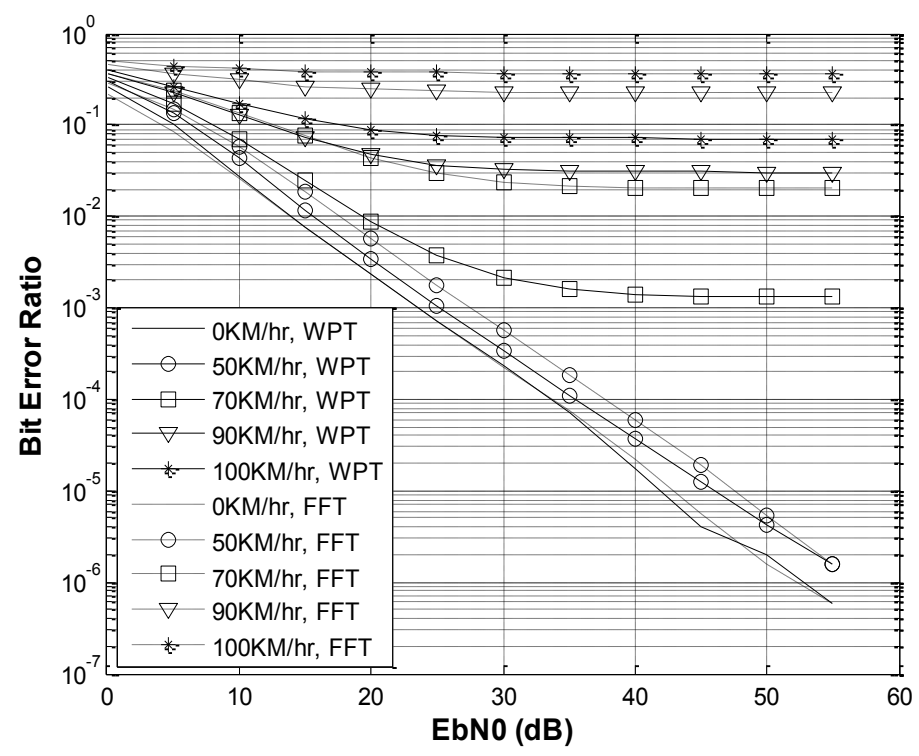

Fig. 4. Comparison of FFT-OFDM and wavelet-OFDM at $2.6 \mathrm{GHz}$ for SISO Systems

Results in Figure 4 show the consistence of waveletOFDM robustness over doubly selective transmission. Meanwhile, the results reveal that the $2.6 \mathrm{GHz}$ has poorer performance than the $800 \mathrm{~Hz}$ operating carrier frequency since the fading bandwidth of the channel increases with the operating carrier frequency.

\section{B. $M I M O-O F D M$}

In MIMO-OFDM case, Figure 5 depicts the performance of FFT-OFDM and wavelet-OFDM for a MISO System for different mobile speeds.

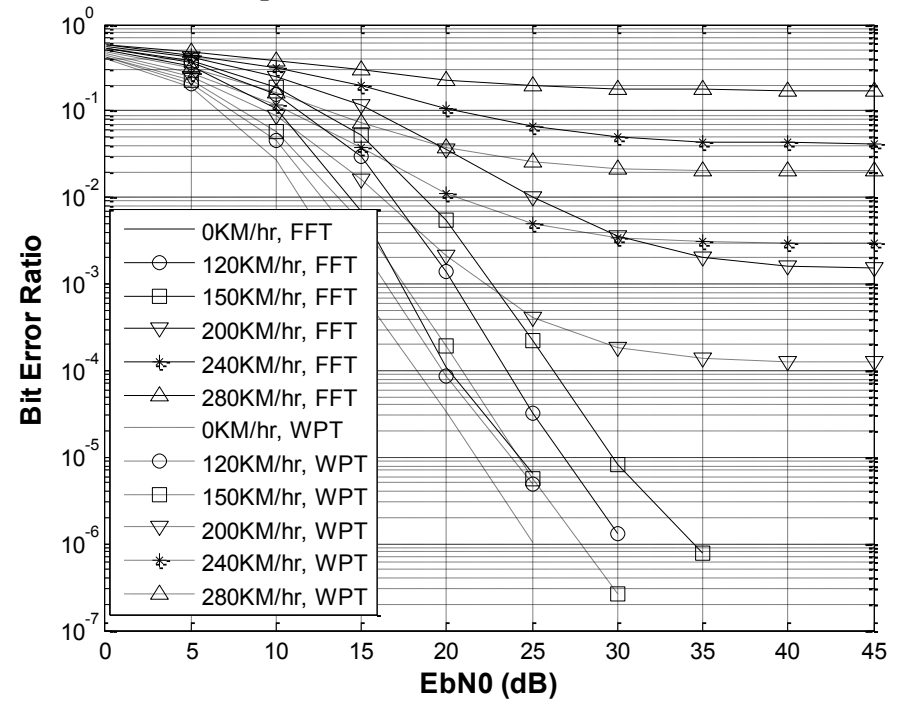

Fig. 5. Comparison of a $2 \times 1$ FFT-OFDM and wavelet-OFDM Systems at $800 \mathrm{MHz}$

It is obvious that the wavelet-OFDM outperformed the FFT-OFDM for all speeds. In the next result (Figure 6), it is shown that the result in Figure 5 is improved for a $2 \times 2$ MIMO system. We conjecture that this result in Figures 5 and 6 may be consistent for $2 \times 1,2 \times 2$ or even higher order MIMO configurations above $2.6 \mathrm{GHz}$ operating frequency, with wavelet-OFDM dominating FFT-OFDM in performance.

In general, the degree at which the OFDM signal shifts from the carrier (or equivalently centre frequency in the baseband) frequency, $f_{c}$, by Doppler shift depends on the property of the signal volunteered by the baseband transform. The Doppler Effect leads to the loss in orthogonality of the sub-channels of the OFDM system. Wavelet transforms the signal to possess time and frequency diversities whereas the FFT provides frequency-selectivity (diversity) only. The robustness of the signal against the Doppler shift is then dependent on characterizing signal property nominated by the baseband transform used in the signal multiplexing.

\section{CONCLUSION}

Future generation wireless communication design of radio air-interface such as the OFDM was presented for two different design kernels - fast Fourier transform and wavelet packet transform. Both OFDM systems were investigated for different mobile speeds which account for varying Doppler frequencies effect in high speed wireless communications typical of real-life scenarios. OFDM converts frequencyselective channel into flat fading channel thus very robust in frequency-selective transmission. However, the FFT-OFDM scheme was observed to be sub-optimal compared to the wavelet transform for OFDM design for high speed mobiles. Results reveal that the wavelet-OFDM has very robust ability in repelling the inter-carrier interference volunteered by fast fading channels due to Doppler Effect than the FFT-OFDM. 
Thus, the wavelet transform can be a good candidate for the seamless broadband network radio air-interface in future wireless communications.

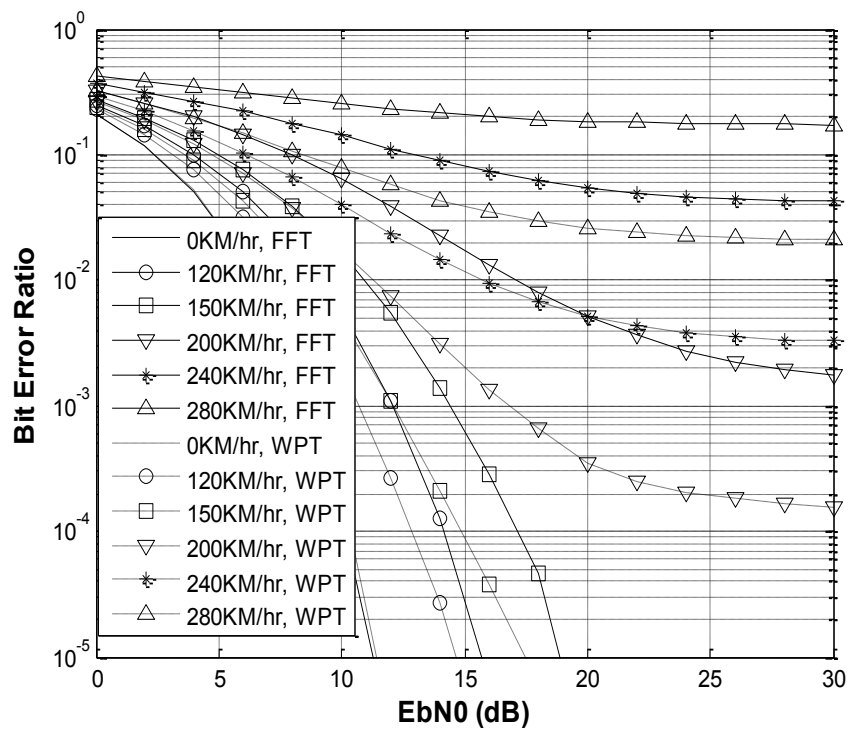

Fig. 6. Comparison of a 2x2 FFT-OFDM and wavelet-OFDM Systems at $800 \mathrm{MHz}$

\section{REFERENCES}

[1] G. L. Stuber, J. R. Barry, S. W. Mclaughlin, Y. Li, M. A. Ingram, and T. G. Pratt, "Broadband MIMO-OFDM wireless communications," Proceedings of the IEEE, vol. 92, pp. 271-294, 2004.

[2] H. Yang, "A road to future broadband wireless access: MIMO-OFDMbased air interface," IEEE Communications Magazine, vol. 43, pp. 5360, 2005 .

[3] S. Parkvall, E. Dahlman, A. Furuskar, Y. Jading, M. Olsson, S. Wanstedt, and K. Zangi, "LTE-advanced-evolving LTE towards IMTadvanced," in IEEE 68th Vehicular Technology Conference, 2008. VTC 2008-Fall. 2008, pp. 1-5.

[4] P. E. Mogensen, T. Koivisto, K. I. Pedersen, I. Z. Kovacs, B. Raaf, K. Pajukoski, and M. J. Rinne, "LTE-advanced: the path towards gigabit/s in wireless mobile communications," in Wireless VITAE 2009. 1st International Conference on Wireless Communication, Vehicular Technology, Information Theory and Aerospace \& Electronic Systems Technology, 2009. 2009, pp. 147-151.

[5] G. Tingting and S. Bin, "A high-speed railway mobile communication system based on LTE," in 2010 International Conference On Electronics and Information Engineering (ICEIE), 2010, pp. V1-414-V1-417.

[6] A. Omri, R. Hamila, M. O. Hasna, R. Bouallegue, and H. Chamkhia, "Estimation of highly Selective Channels for Downlink LTE MIMOOFDM System by a Robust Neural Network," JUSPN, vol. 2, pp. 31-38, 2011.

[7] A. Ghosh, R. Ratasuk, B. Mondal, N. Mangalvedhe, and T. Thomas, "LTE-advanced: next-generation wireless broadband technology [Invited Paper]," IEEE Wireless Communications, vol. 17, pp. 10-22, 2010.

[8] J. Wannstrom. (2012). LTE-Advanced. Available: http://www.3gpp.org/lte-advanced (Accessed on Sept. 19, 2013)

[9] Cisco, "802.11ac: The Fifth Generation of Wi-Fi Technical White Paper," ed, Aug. 2012.

[10] T. Bao and L. Yongling, "Improved space-time-frequency block code for MIMO-OFDM wireless communications," in 2012 IEEE International Conference on Signal Processing, Communication and Computing (ICSPCC), 2012, pp. 538-541.

[11] W. Zhang, X. Xiang-Gen, and K. Ben Letaief, "Space-time/frequency coding for MIMO-OFDM in next generation broadband wireless systems," IEEE Wireless Communications, vol. 14, pp. 32-43, 2007.
[12] H. Bolcskei, "MIMO-OFDM wireless systems: basics, perspectives, and challenges," IEEE Wireless Communications, vol. 13, pp. 31-37, 2006.

[13] H. Bolcskei and A. J. Paulraj, "Space-frequency coded broadband OFDM systems," in 2000 IEEE Wireless Communications and Networking Confernce, 2000. WCNC. 2000, pp. 1-6.

[14] H. Bolcskei and A. J. Paulraj, "Space-frequency codes for broadband fading channels," in 2001 IEEE International Symposium on Information Theory, 2001. Proceedings. 2001, p. 219.

[15] Z. Liu, Y. Xin, and G. B. Giannakis, "Space-time-frequency coded OFDM over frequency-selective fading channels," IEEE Transactions on Signal Processing, vol. 50, pp. 2465-2476, 2002.

[16] O. O. Anoh, N. T. Ali, R. Abd-Alhameed, S. M. Jones, and Y. A. Dama, "On the performance of DWT and WPT modulation for multicarrier systems," in 2012 IEEE 17th International Workshop on Computer Aided Modeling and Design of Communication Links and Networks (CAMAD), 2012, pp. 348-352.

[17] A. Jamin and P. Mähönen, "Wavelet packet modulation for wireless communications," Wireless Communications and Mobile Computing, vol. 5, pp. 123-137, 2005.

[18] H. M. Ozaktas, B. Barshan, D. Mendlovic, and L. Onural, "Convolution, filtering, and multiplexing in fractional Fourier domains and their relation to chirp and wavelet transforms," JOSA A, vol. 11, pp. 547-559, 1994.

[19] B. Negash and H. Nikookar, "Wavelet-based multicarrier transmission over multipath wireless channels," Electronics Letters, vol. 36, pp. 1787$1788,2000$.

[20] B. Negash and H. Nikookar, "Wavelet based OFDM for wireless channels," in IEEE VTS 53rd Vehicular Technology Conference, 2001. VTC 2001 Spring. 2001, pp. 688-691.

[21] Y. Zhang and S. Cheng, "A novel multicarrier signal transmission system over multipath channel of low-voltage power line," IEEE Transactions on Power Delivery, vol. 19, pp. 1668-1672, 2004.

[22] F. Fazel and H. Jafarkhani, "Quasi-orthogonal space-frequency and space-time-frequency block codes for MIMO OFDM channels," IEEE Transactions on Wireless Communications, vol. 7, pp. 184-192, 2008.

[23] D. H. Nguyen and H. H. Nguyen, "Diversity and Coding Gains of Space-Time-Frequency Coded MIMO-OFDM," in IEEE, 2008 Vehicular Technology Conference, 2008. VTC Spring 2008., pp. 518523.

[24] H. Jafarkhani, "A quasi-orthogonal space-time block code," IEEE Transactions on Communications, vol. 49, pp. 1-4, 2001.

[25] [25] S. M. Alamouti, "A simple transmit diversity technique for wireless communications," IEEE Journal on Selected Areas in Communications, vol. 16, pp. 1451-1458, 1998.

[26] W. Su, Z. Safar, M. Olfat, and K. R. Liu, "Obtaining full-diversity space-frequency codes from space-time codes via mapping," IEEE Transactions on Signal Processing, vol. 51, pp. 2905-2916, 2003.

[27] D.-B. Lin, P.-H. Chiang, and H.-J. Li, "Performance analysis of twobranch transmit diversity block-coded OFDM systems in time-varying multipath Rayleigh-fading channels," IEEE Transactions on Vehicular Technology, vol. 54, pp. 136-148, 2005.

[28] W. Zhang, X.-G. Xia, and P.-C. Ching, "High-rate full-diversity spacetime-frequency codes for broadband MIMO block-fading channels," IEEE Transactions on Communications, vol. 55, pp. 25-34, 2007.

[29] K. O. O. Anoh, R. A. Abd-alhameed, J. M. Noras, and S. M. R. Jones, "Wavelet Packet Transform Modulation for Multiple Input Multiple Output Applications," IJCA, vol. 63 - Number 7, pp. 46 - 51, 2013.

[30] K. O. O. Anoh, R. A. Abd-Alhameed, and S. M. R. Jones, "An Investigation of FrFT Modulation and FFT-OFDM for MIMO Applications," presented at the 14th Annual Post Graduate Symposium on Convergence of Telecommunications, Networking and Broadcasting, Liverpool, UK, 24 - 25th June, , 2013.

[31] G. L. Stüber, Principles of mobile communication: Springer, 2011.

[32] T. Wang, J. G. Proakis, E. Masry, and J. R. Zeidler, "Performance degradation of OFDM systems due to Doppler spreading," IEEE Transactions on Wireless Communications, vol. 5, pp. 1422-1432, 2006. 